\title{
ROLE: OF STRAIN ON THRESHOLD CURRENT, MODAL PURITY AND AUGER PROCESSES IN STRAINED QUANTUM WELL LASERS
}

\author{
John P. Loehr and Jasprit Singh \\ Center for High Frequency Microelectronics \\ Department of Electrical Engineering and Computer Science \\ University of Michigan \\ Ann Arbor, Michigan 48109-2122
}

(Received 30 July 1990)

\begin{abstract}
We present numerical calculations of material gain and threshold current density in compressively strained quantum well lasers grown on GaAs and InP. The valence bandstructure is obtained from a $4 \times 4 \mathrm{k} \cdot \mathbf{p}$ Hamiltonian that includes the effects of strain. Calculation of the spontaneous emission rate and optical material gain proceeds directly from the bandstructure and we extract the threshold current density from the emission rate. We find that incorporating $\sim 2 \%$ misfit strain reduces the threshold current density by $50 \%$ in the GaAs system and by $30 \%$ in the InP system. We calculate the hole masses in the presence of strain with a $6 \times 6 \mathrm{k} \cdot \mathbf{p}$ Hamiltonian and use them to determine the effect of strain on Auger processes.
\end{abstract}

The performance of quantum well lasers can be dramatically improved by incorporating biaxial strain into the well. The strain serves to reduce the threshold current and to improve the time response of these devices as theoretical and experimental studies have shown. ${ }^{1-4}$ Because all of the performance enhancements resulting from strain and quantization are due to changes in the bandstructure, it is important to have a modelling tool which will calculate the device parameters directly from the bands; in this paper we present the results of such a model. We calculate the effects of strain on the quantum well bandstructure and use the results to compute the optical properties of the device and the associated threshold current. In particular, we evaluate the spontanteous emission directly from the bandstructure using Fermi statistics. We then use this model to optimize a multiquantum well laser for low threshold current density and present results for the optimized structure. Lastly, we calculate the effect of strain on the valence bandgaps and masses and use the results to predict the strain dependence of the CHHS Auger rates.

To accurately model long-wavelength optical transitions in III-V semiconductors it is sufficient to include only the conduction band, heavy hole $\left(\mathrm{HH},\left|\frac{3}{2}, \pm \frac{3}{2}\right\rangle\right)$, and light hole ( $\left.\mathrm{LH},\left|\frac{3}{2}, \pm \frac{1}{2}\right\rangle\right)$ states; the split-off (SO, $\left.\left|\frac{1}{2}, \pm \frac{1}{2}\right\rangle\right)$ band does not participate in low energy transi- tions. We describe the valence band edge with the $4 \times 4$ $\mathbf{k} \cdot \mathbf{p}$ Hamiltonian. ${ }^{5}$ In this formalism, the hole wavefunction is given by

$$
\left\langle\mathbf{r}_{h} \mid m, \mathbf{k}\right\rangle=\frac{e^{i \mathbf{k} \cdot \boldsymbol{\rho}_{h}}}{2 \pi} \sum_{\nu} g_{m}^{\nu}\left(\mathbf{k}, z_{h}\right) U_{\nu}^{h}\left(\mathbf{r}_{h}\right)
$$

where $\mathbf{k}$ is the in-plane two-dimensional wavevector, $\boldsymbol{\rho}_{h}$ is the in-plane radial coordinate, $z_{h}$ is the coordinate in the growth direction, the $U_{\nu}^{h}$ are the zone center Bloch functions having spin symmetry $\nu$, and $m$ is a subband index. The envelope functions $g_{m}^{\nu}\left(\mathbf{k}, z_{h}\right)$ and subband energies $E_{m}^{h}(\mathbf{k})$ satisfy the Luttinger-Kohn equation in a quantum well. ${ }^{6}$ The effect of strain is incorporated via a splitting $\delta$ between the light and heavy hole diagonal elements. For $\operatorname{In}_{x} \mathrm{Ga}_{1-x}$ As grown on $\mathrm{GaAs}$ and $\mathrm{In}_{0.53+x} \mathrm{Ga}_{0.47-x}$ As grown on InP it is given (in $\mathrm{eV}$ ) by $\delta=-5.966 \epsilon$, where the lattice mismatch $\epsilon$ is related to the excess In composition $x$ by $\epsilon=-(0.07) x{ }^{7}$ This splitting reduces the off-diagonal mixing between the $\mathrm{HH}$ and LH states and changes the effective masses of the holes.

Figure 1 shows the hole dispersion curves for a $50-\AA$ a) $\mathrm{GaAs} / \mathrm{Al}_{0.3} \mathrm{Ga}_{0.7} \mathrm{As}$ and b) $\mathrm{In}_{0.2} \mathrm{Ga}_{0.8} \mathrm{As} / \mathrm{Al}_{0.3} \mathrm{Ga}_{0.7} \mathrm{As}$ (on GaAs) quantum well. As can be seen quite clearly, the strained structure exhibits a much sharper curvature representing a much lower band edge mass. The 

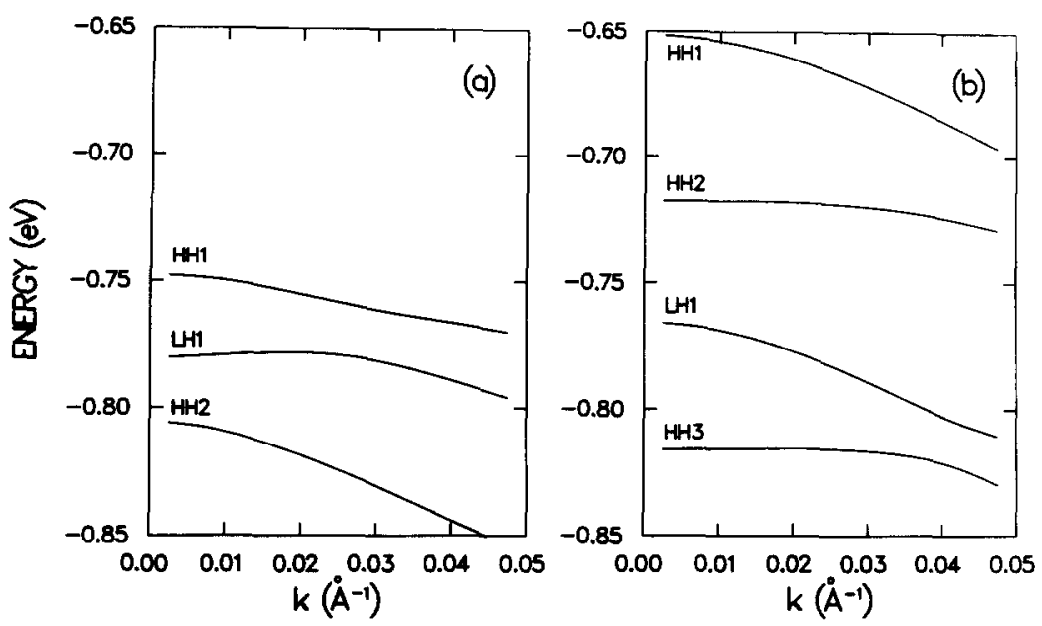

Fig. 1 Calculated valence bandstructure along the [100] direction in a $50-\AA$ a) $\mathrm{GaAs} / \mathrm{Al}_{0.3} \mathrm{Ga}_{0.7} \mathrm{As}$ (on $\mathrm{GaAs}$ ) and b) $\mathrm{In}_{0.12} \mathrm{Ga}_{0.8} \mathrm{As} / \mathrm{Al}_{0.3} \mathrm{Ga}_{0.7} \mathrm{As}$ (on $\mathrm{GaAs}$ ) quantum well.

hole masses can be decreased by up to a factor of 3 by increasing the In content. ${ }^{6}$ We also note that strain forces the LH subbands far below the bandedge which makes their occupation negligible at room temperature.

In the conduction band, the strain serves only to shift the band edge and slightly change the effective mass. ${ }^{6}$ Hence, we use a simple scalar effective mass theory and write the electron state $|n, \mathbf{k}\rangle$ as

$$
\left\langle\mathbf{r}_{e} \mid n, \mathbf{k}\right\rangle=\frac{e^{i \mathbf{k} \cdot \boldsymbol{\rho}_{e}}}{2 \pi} f_{n}\left(z_{e}\right) U_{\sigma}^{e}\left(\mathbf{r}_{e}\right)
$$

The envelope functions $f_{n}\left(z_{e}\right)$ and subband energies $E_{n}^{e}(\mathbf{k})$ satisfy the effective mass Schrodinger equation which we solve by using finite difference methods.

We now calculate the optical material gain directly from the bandstructure using the Fermi golden rule, the result is tinuum excitons, we multiply Eq. (3) by a quasi twodimensional Coulomb enhancement factor taken from Ref. 8. To get the total material gain $g$ we sum over all subbands $n, m$ and integrate against a broadening function. The optical matrix element $\mathbf{P}_{n m}^{\sigma}(\mathbf{k})$ is obtained by multiplying the overlap integral of the electron and hole wavefunctions by the momentum matrix elements, i.e.

$$
\mathbf{P}_{n m}^{\sigma}(\mathbf{k})=\sum_{\nu} \int f_{n}^{*}(z) g_{m}^{\nu}(\mathbf{k}, z) d z\left\langle U_{\sigma}^{e}|\mathbf{p}| U_{\nu}^{h}\right\rangle
$$

We evaluate the monentum matrix elenents

$\left\langle U_{\sigma}^{e}|\mathbf{p}| U_{\nu}^{h}\right\rangle$ as in Ref. 8. Note that $\hat{x}$-polarized light (TE mode) couples 3 times more strongly to $\mathrm{HH}$ states

$g_{n m}(\hbar \omega)=\frac{4 \pi^{2} e^{2} \hbar}{n_{0} c m_{0}^{2} W \hbar \omega} \frac{1}{(2 \pi)^{2}} \int d \mathbf{k} \sum_{\sigma}\left|\hat{\boldsymbol{\epsilon}} \cdot \mathbf{P}_{n m}^{\sigma}(\mathbf{k})\right|^{2} \delta\left(E_{n}^{\mathbf{e}}(\mathbf{k})-E_{m}^{h}(\mathbf{k})-\hbar \omega\right)\left[f^{e}\left(E_{n}^{e}(\mathbf{k})\right)-f^{h}\left(E_{m}^{h}(\mathbf{k})\right)\right]$

where $n_{0}$ is the index of refraction, $W$ is the quantum well width, $c$ is the speed of light, $\hat{\epsilon}$ is the polarization of the light, $\hbar \omega$ is the photon energy, and $f^{e}$ and $f^{h}$ are the distribution functions for electrons in the conduction and valence bands, respectively. We take $f^{e}$ and $f^{h}$ to be Fermi functions and solve numerically for the quasi-Fermi levels as a function of carrier injection. To account for the effects of transitions resulting from con- than to LH states and that $\hat{z}$-polarized light (TM mode) couples exclusively to the LH states.

In order to determine the threshold current density we must evaluate the spontaneous emission rate per unit area. This is done by evaluating the dipole transition rate $^{9}$ for the quantum-confined carrier states, giving 


$$
R_{s p}=\int d(\hbar \omega) \frac{4 e^{2} n_{0} \hbar \omega}{3 m_{0}^{2} c^{3} \hbar^{2}} \frac{1}{(2 \pi)^{2}} \sum_{n m} \int d \mathbf{k} \sum_{\sigma}\left|\mathbf{P}_{n m}^{\sigma}(\mathbf{k})\right|^{2} \delta\left(E_{n}^{e}(\mathbf{k})-E_{m}^{h}(\mathbf{k})-\hbar \omega\right)\left[f^{e}\left(E_{n}^{e}(\mathbf{k})\right)\right]\left[1-f^{h}\left(E_{m}^{h}(\mathbf{k})\right)\right]
$$

The current density can then be determined in the approximation that, at threshold, all of the injected current is converted into spontaneous emission. For a multiquantum well structure with $N$ identical wells this gives

$$
J=e N K_{s p} .
$$

If the injected electrons and holes obeyed Boltzmann statistics the current would be proportional to the squarc of the injected carrier density $n$. At high injections, however, we must use Fermi statistics and the current falls off from the $n^{2}$ curve.

The laser parameters discussed in the remainder of this paper are for a separate optical confinement heterostructure laser. We will not deal explicitly with the confinement of the optical mode, but will focus instead on the properties that arc directly related to the band structure and the associated carrier concentrations in the active well region. We assume that the optical confinement factor $\Gamma$ is given by

$$
\Gamma=N \gamma W
$$

where $N$ is the number of quantum wells used in the multiquantum well structure, $W$ is the width of each individual quantum well, and $\gamma$ is the optical confinement per unit width of quanturn well. We assume the typjcal values for $\gamma$ of $2.5 \times 10^{-4} \AA^{-1}$ for GaAs-based and $2.0 \times 10^{-4} \AA^{-1}$ for InP-based lasers; $\gamma$ is smaller in the InP system because of the longer optical wavelength.

To calculate the value of the material gain required for lasing we require that at threshold the modal gain $\Gamma g$ balance the internal losses, i.e.

$$
\Gamma g=\frac{1}{L} \ln \frac{1}{R}+\alpha .
$$

Here $g$ is the material gain, $L$ is the cavity length, $R$ is the mirror reflectivity, and $\alpha$ is the nonradiative absorption loss per unit length. For our device we assume that $R=.32, L=300 \mu \mathrm{m}$, and $\alpha=10 \mathrm{~cm}^{-1}$ which forces $\Gamma g=48 \mathrm{~cm}^{-1}$.

Note that $\Gamma g$, the modal gain, does not depend explicitly on $W$, the quantum well width. It depends only indirectly on $W$ in that changing the width affects the separation between the subbands and the overlap between electrons and holes. We now use our model to optimize a multiquantum well structure for low threshold current density.

The parameters that one has control over are i) compostion of the quantum wells (strain) ji) width of the quantum wells and iii) number of quantum wells and the separation between them; we will choose each design parameter to minimize the threshold current den- sity. Population inversion can be maximized, for a given carrier density, by minimizing the bandedge density of states and thus increasing the separation between the electron and hole quasi-Fermi levels. Applying biaxial compressive strain will reduce the $\mathrm{HH}$ effective mass and will increase the separation between the $\mathrm{HH}$ and $\mathrm{LH}$ sub bands; both of these effects reduce the valence bandedge density of states. The separation between subbands can also be increased by narrowing the quantum wells. Hence, to maximize the inversion it is advantageous to use large strains and narrow quantum wells. However, we note that the gain term also depends upon the overlap $\mathbf{P}_{n m}^{\sigma}(\mathbf{k})$ between the electrons and holes. Since the electron mass is much smaller than the hole masses, this function will decrease at small well sizes. Hence, a compromise must be chosen between inversion and overlap to maximize the gain as a function of well size while remaining below the critical thickness. Another design parameter is the number of quantum wells grown and the spacing between the quantum wells. If the quantum wells are very close together (coupled) then the mixing between the wells gives a larger bandedge density of states and poor inversion. Placing $N$ wells farther apart in a multiquantum well structure will give better performance. The optimal number of wells will depend on the variation with carrier density of the spontaneous emission rate and material gain. For the losses we have chosen, our calculations show that a single quanturn well will require the lowest threshold current density.

Taking into account all of the above considerations, we find that the optimal well widths are $W=50-\AA$ for GaAs-based and $W=80-\stackrel{A}{A}$ for InF-based lasers. These well sizes are comparable to those which are experimentally found to give low threshold devices. ${ }^{10}$ The wider well size necessary for the InP system is due to the very small electron mass which results in a small overlap function at smaller well sizes. Hence, the primary constraint on well size for the InP system is that it be below the critical thickness for the strained layer.

We note that as excess In is added to the quantum well the bandgap changes and thus the emission frequency will change; we have neglected this effect in our calculations for the InP-based system. If the emission frequency is to be fixed, one may need to add excess $\mathrm{Al}$ in the well (i.e. use quaternary wells). Since the changes in the bandstructure are primarily strain related, this will not change the results reported here.

We will now present our results for the optimized device structures. In Fig. 2 we show the TE mode ( $\dot{x}$ polarized) material gain in the GaAs system at $300 \mathrm{~K}$ for various carrier concentrations. Figure 2(a) shows the lattice-matched results and Fig. 2(b) gives the re- 

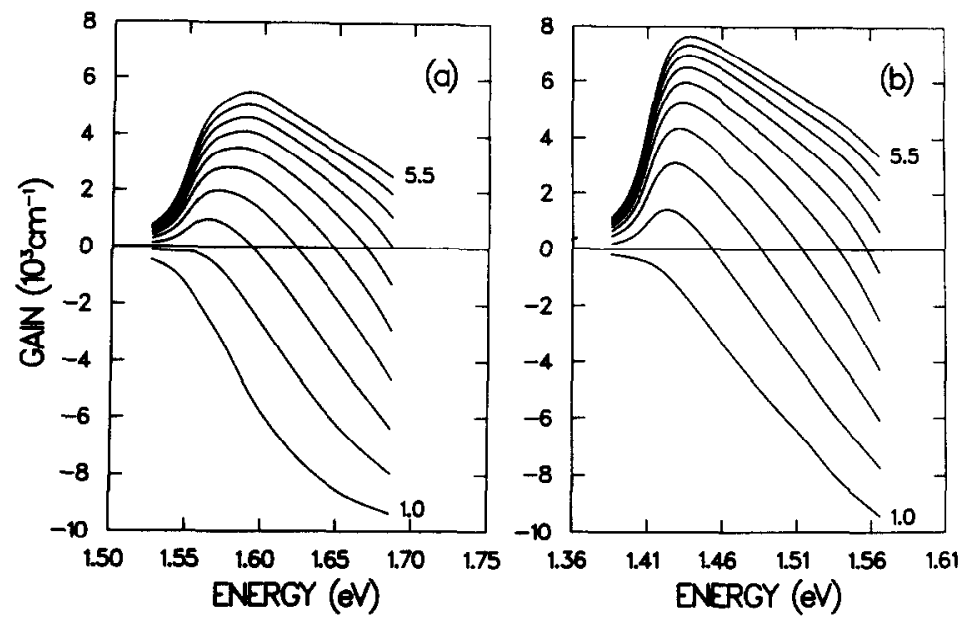

Fig. 2 TE mode material gain in a $50-\AA$ a) GaAs/

$\mathrm{Al}_{0.3} \mathrm{Ga}_{0.7} \mathrm{As}$ (on GaAs) and b) $\mathrm{In}_{0.12} \mathrm{Ga}_{0.8} \mathrm{As} /$

$\mathrm{Al}_{0.3} \mathrm{Ga}_{0.7} \mathrm{As}$ (on $\mathrm{GaAs}$ ) quantum well at $300 \mathrm{~K}$ for vari-

ous carrier injections. Injections are given in

$\left(10^{12}\right.$ carriers $\left./ \mathrm{cm}^{2}\right)$ in steps of $.5 \times 10^{12} / \mathrm{cm}^{2}$.
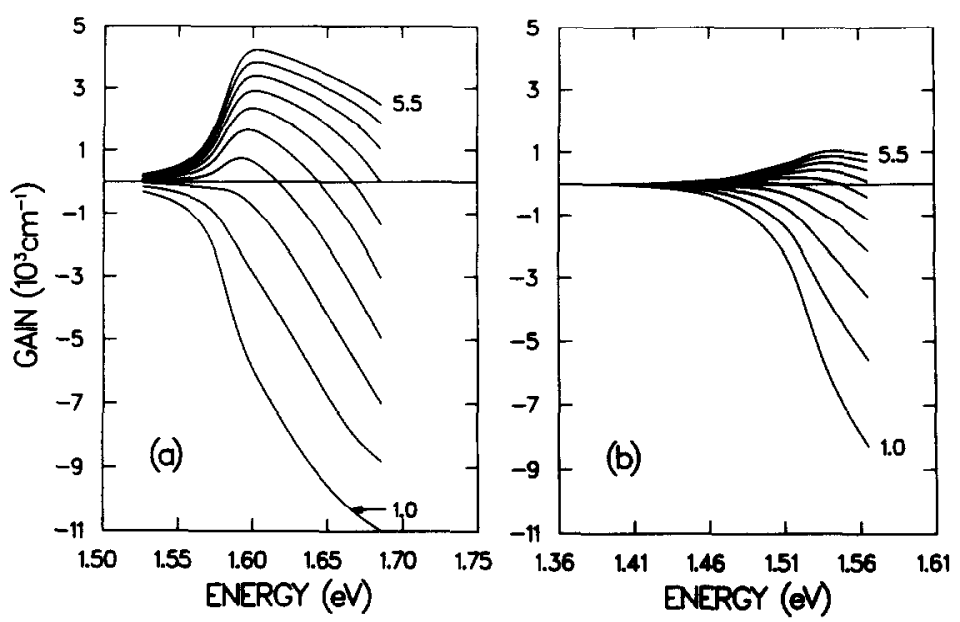

Fig. 3 TM mode material gain in a $50-\AA$ a) GaAs/

$\mathrm{Al}_{0.3} \mathrm{Ga}_{0.7} \mathrm{As}$ (on GaAs) and b) $\mathrm{In}_{0.12} \mathrm{Ga}_{0.8} \mathrm{As} /$

$\mathrm{Al}_{0.3} \mathrm{Ga}_{0.7} \mathrm{As}$ (on GaAs) quantum well at $300 \mathrm{~K}$ for vari-

ous carrier injections. Injections are given in

$\left(10^{12}\right.$ carriers $\left./ \mathrm{cm}^{2}\right)$ in steps of $.5 \times 10^{12} / \mathrm{cm}^{2}$.

sults with $20 \%$ In added to the well. We see that the strained well exhibits much higher gain for the same carrier injections because of the smaller heavy hole mass. In contrast, Fig. 3 shows the TM mode ( $\hat{z}$-polarized) gain for the same structures. Here the strain pushes the light hole subbands far below the band edge which greatly reduces their occupation. Since the TM mode gain results exclusively from light hole transitions, it is much reduced by the application of strain. The material gain $g$ required for TE mode lasing is obtained from Eq. (8) 


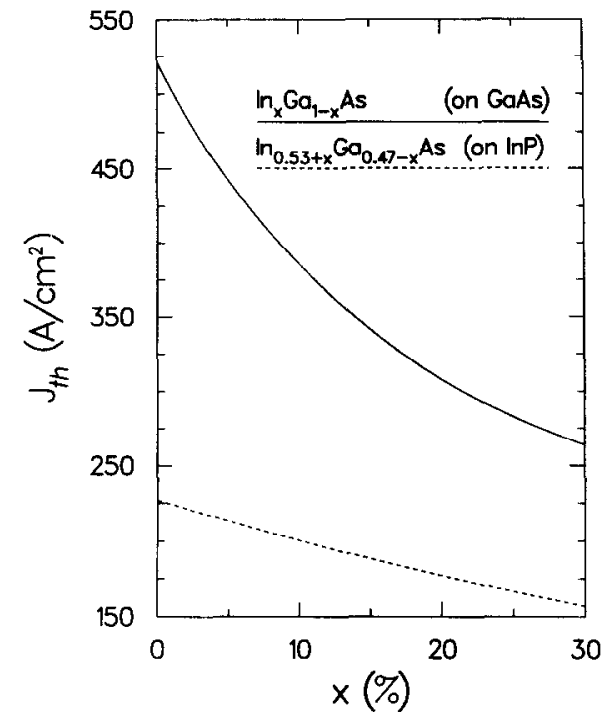

Fig. 4 Threshold current density verses (x).

and the corresponding carrier concentration can be read from Fig. 3. Evaluating the spontaneous emission rate at this carrier concentration gives the threshold current density. Figure 4 shows the threshold current density as a function of In added for the GaAs- and InP-based systems. We see that adding $30 \%$ excess In (corresponding to $2.1 \%$ strain) reduces the threshold by $50 \%$ in the GaAs system and by $30 \%$ in the InP system. The InP system exhibits a smaller improvement with strain because the electron effective mass is lower. Hence, the quasi-Fermi level split is, to a greater extent, dominated by the electron quasi-Fermi level. Since the effect of strain is primarily to shift the hole quasi-Fermi level we see a lesser performance improvement as the strain increases in the InP system.

We calculate the effect of strain on Auger processes by modifying the threshold conditions of Beattie and Landsberg ${ }^{11}$ to account for different masses in the initial and final states. Because of the large spin-orbit splitting in InGaAs, we assume that transitions from the heavy holes to the split-off holes (CHHS) will be the dominant Auger mechanism. We use the $6 \times 6 \mathrm{k} \cdot \mathrm{p}$ Hamiltonian and the deformation potential theory to calculate the heavy hole, light hole, and split-off bands in the presence of strain; ${ }^{12}$ the bands are calculated for bulk material. We find that the heavy and light hole masses change dramatically with the application of strain, as described above, but that the split-off mass changes very little. The bandgap and the split-off gap, however, change signficantly and we use them in the threshold conditions to obtain the electron $\left(E^{e}\right)$ and hole $\left(E^{h}\right)$ energies for the

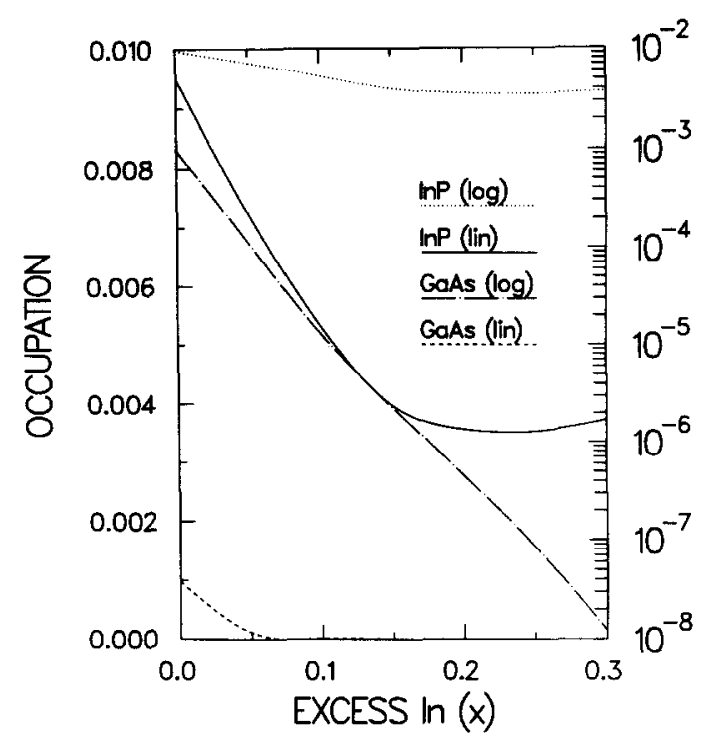

Fig. 5 Occupation factor $\left[1-f^{h}\left(E^{h}\right)\right]^{2}\left[f^{e}\left(E^{e}\right)\right]$ for $\mathrm{In}_{x} \mathrm{Ga}_{1-x} \mathrm{As}$ (on GaAs) and $\mathrm{In}_{\mathbf{0 . 5 3 + x}} \mathrm{Ga}_{0.47-x}$ As (on InP) as function of $(x)$ on a linear and $\log$ scale.

most probable transition. We then compute the probability that the initial state is full and the final state is empty by evaluating the Fermi functions $f^{e}$ and $f^{h}$ at the lasing carrier density. In Fig. 5 we plot the resulting occupation factor of $\left[1-f^{h}\left(E^{h}\right)\right]^{2}\left[f^{e}\left(E^{e}\right)\right]$ as a function of the excess In mole fraction. We see that adding $30 \%$ In reduces the occupation by 5 orders of magnitude for devices grown on $\mathrm{GaAs}$ and by a factor of 3 for those based on InP; the Auger recombination rates should exhibit a sinilar behavior.

In conclusion, we have calculated the optical properties and threshold current density of a strained quantum well laser directly from the bandstructure. We find that strain signficantly reduces the threshold current density and Auger recombination rates and dramatically increases the ratio of TE to TM mode material gain. The structure requiring the lowest threshold current density is found to be a single quanturn well that is $\sim 50$ - $\AA$ wide for devices grown on GaAs and $\sim 80$ - $\AA$ wide for those grown on InP. The model presented is very general and could be extended to predict the time response of these devices.

Acknowledgement - The authors thank J. M. Hinckley for the use of his programs to calculate valence band masses and bandgaps as a function of strain. This work was supported by a grant from the Army Research Office, URI program. 


\section{References}

1. W. D. Laidig, P. J. Caldwell, Y. F. Lin and C. K. Peng, Appl. Phys. Lett. 44, 653 (1984).

2. G. Feak, D. Nichols, J. Singh, J. Loehr, J. Pamulapati, P. Bhattacharya and D. Biswas, Proceedings of the IEEE Cornell Conference on Advanced Concepts in High Speed Semiconductor Devices and Circuits, p. 362, 1989.

3. A. Ghiti, W. Batty, U. Ekenberg and E. P. O'Reilly, SPIE vol. 861 Quantum Wells and Superlattices in Optoelectronic Devices and Integrated Optics, p. $96,1987$.

4. D. Ahn and S. L. Chuang, IEEE J. Quantum Electron. QE-24, 2400 (1988).
5. J. M. Luttinger and W. Kohn, Phys. Rev. 97, 869 (1955).

6. M. Jaffe and J. Singh, J. Appl. Phys. 65, 329 (1989).

7. H. Kato, N. Iguchi, S. Chika, M. Nakayama and N. Sano, J. Appl. Phys. 52, 588 (1986).

8. G. D. Sanders and Y. C. Chang, Phys. Rev. B 35, 1300 (1987).

9. J. J. Sakurai, Advanced Quantum Mechanics. p. 43, New York: Addison-Wesley, 1967.

10. C. A. Wang, Integrated Photonics Research Conference, Hilton Head, South Carolina, March 1990.

11. A. R. Beattie and P. T. Landsberg, Proc. Roy. Soc. A 249, 16 (1959).

12. J. M. Hinckley and J. Singh, to be published in Phys. Rev. B. 